\title{
Metabolic characterization of the bovine blastocyst, inner cell mass, trophectoderm and blastocoel fluid
}

\author{
N. Gopichandran and H. J. Leese* \\ Department of Biology (Area 3), University of York, PO Box 373, York YO10 5YW, UK
}

The formation of a viable blastocyst is dependent upon the establishment of a correct inner cell mass (ICM):trophectoderm cell ratio but little is known about the metabolism of the two cell populations or about the composition of blastocoel fluid. In this study, the metabolism of intact bovine blastocysts, isolated ICM and trophectoderm was examined in terms of glucose and pyruvate uptake, lactate production, and amino acid consumption or production. The concentration of these nutrients in blastocoel fluid was also determined. The metabolism of glucose, pyruvate and lactate differed significantly between the isolated ICM and trophectoderm. Isolated trophectoderm had a higher pyruvate $(P<0.001)$ and lower glucose $(P<0.05)$ consumption, and higher lactate production $(P<0.05)$ than did ICM. The consumption or production of amino acids by ICM and trophectoderm also differed, with the trophectoderm displaying a higher turnover (the sum of production and consumption). The ICM and trophectoderm both depleted arginine, aspartate and leucine, whereas the production of alanine was consistent. Isolated ICM depleted a further six amino acids, which appeared during trophectoderm culture; the reverse trend was observed for the remaining amino acids. The concentration of lactate in blastocoel fluid was significantly higher than in synthetic oviductal fluid supplemented with amino acids and BSA (SOFaaBSA; $P<0.05$ ). However, glucose $(P<0.05)$ and pyruvate $(P<0.001)$ concentrations were both lower. Aspartate, glutamate, glycine, alanine and tryptophan were present at significantly higher concentrations in blastocoel fluid than in SOFaaBSA, whereas threonine and asparagine concentrations were significantly lower. The metabolism of composite blastocysts, obtained by summing the consumption and production profiles of the ICM and trophectoderm, and taking into account their respective number of cells, was higher than that of intact blastocysts, indicating that upon isolation of the two cell populations there may be disruption to paracrine interactions or the onset of culture-induced cellular stress or both.

\section{Introduction}

Development of mammalian preimplantation embryos involves the formation of the blastocyst in which the outer trophectoderm cells surround the blastocoel containing the inner cell mass (ICM). The trophectoderm is the first epithelium to form during mammalian development and gives rise to extraembryonic structures, whereas the ICM develops principally into the embryo proper.

The cells of the trophectoderm are polarized, with a microvillous apical surface and a relatively smooth basolateral surface that borders the blastocoel fluid (Ducibella and Anderson, 1975), whereas those of the ICM are apolar and devoid of microvilli (Pratt, 1985). The trophectoderm acts as a selective barrier to the movement of solutes into and out of the blastocoel; in particular, the $\mathrm{Na}^{+} / \mathrm{K}^{+}$-ATPase localized to the basolateral and apical membranes of trophectoderm cells transports $\mathrm{Na}^{+}$into the blastocoel cavity, with water following osmotically

*Correspondence

Email: hjl1@york.ac.uk
(Biggers et al., 1988; Watson and Barcroft, 2001). Regulation of blastocoel fluid formation, and hence the provision of nutrients for the ICM, is determined by the transporting activity of the trophectoderm (Jaszczak et al., 1972; Robinson and Benos, 1991; Brison and Leese, 1993). Differences between trophectoderm and ICM have been examined in terms of gene expression (Fleming and Hay, 1991; Slager et al.,, 1991; Kirchhof et al., 2000), protein synthesis (Handyside and Johnson, 1978) and metabolism (Hewitson and Leese, 1993).

The number of cells in the ICM correlates strongly with normal fetal development (Lane and Gardner, 1997) and the establishment of a correct ICM:trophectoderm cell ratio is considered essential to ensure subsequent embryo viability (Fleming, 1987).

The early cleavage stages of development of bovine preimplantation embryos rely principally on the oxidation of nutrients, specifically pyruvate for energy provision (Thompson et al., 1996). In common with other species (Leese, 1995), there is an increase in glucose consumption and lactate production at compaction (Thompson et al., 1996). Thus, cattle blastocysts rely 
principally on oxidative phosphorylation (86\%) and glycolysis $(14 \%)$ to generate ATP to support cavitation (Rieger et al., 1992; Donnay and Leese 1999).

Amino acids are added to media for culture of early cattle embryos where they serve a variety of physiological functions including: protein synthesis (Epstein and Smith, 1973), energy provision (Partridge and Leese, 1996; Jung et al., 1998), osmoregulation (Baltz, 2001), pH regulation (Baltz, 1993; Edwards et al., 1998), protection against oxidative stress (Lindenbaum, 1973) and removal of ammonium (Leese, 1991; Partridge and Leese, 1996; Donnay and Leese, 1999).

Robinson and Benos (1991) and Hewitson and Leese (1993) hypothesized that the trophectoderm spares glucose for use by ICM in rabbits and mice, respectively. In mice, the two cell lineages differ in their metabolism of glucose and pyruvate, particularly with respect to their reliance on glycolysis. Murine ICM cells have a glycolytic index (the proportion of glucose converted to lactate) of about $100 \%$, which is twice that of the trophectoderm (Hewiston and Leese, 1993). Furthermore, these lineages differ in their glucose storage, as indicated by the higher glycogen content of the trophectoderm compared with the ICM (Edirisinghe et al., 1984).

To the best of our knowledge, there is no information regarding the metabolic properties of the individual cell populations in cattle blastocysts or on the composition of blastocoel fluid. Therefore, the metabolism of isolated bovine ICM and trophectoderm was examined in the present study in terms of glucose and pyruvate uptake, lactate production and amino acid consumption and production. The same measurements have also been obtained for intact blastocysts. This has enabled determination of the extent to which the metabolism of a bovine blastocyst is the sum of its constituent parts.

\section{Materials and Methods}

\section{Collection and maturation of oocytes}

All chemicals were purchased from Sigma (Poole) unless otherwise stated. Ovaries were obtained from a local abattoir and transported to the laboratory in phosphate-buffered saline (PBS) supplemented with $25 \mu \mathrm{l}$ antibiotic-antimycotic $\left(100 \mathrm{ml}^{-1}\right)$ (Gibco Life Technologies, Paisley) at room temperature. Ovarian follicles (3$10 \mathrm{~mm}$ in diameter) were punctured and the follicular fluid was aspirated into Hepes-buffered TCM199 medium containing $50 \mu \mathrm{g}$ kanamycin monosulphate $\mathrm{ml}^{-1}$, $2 \mu \mathrm{g}$ heparin $\mathrm{ml}^{-1}$ (H0777) and $1.5 \mathrm{~g} \mathrm{BSA} \mathrm{l}^{-1}$ (A6003). Cumulus-oocyte complexes (COCs) with several layers of granulosa cells and an evenly shaded cytoplasm were selected, transferred into maturation medium (bicarbonate-buffered TCM199 supplemented with $10 \%$ fetal bovine serum, 0.025 iu FSH/LH (Ferring Pharmaceuticals, Langley), $0.47 \mu \mathrm{g}$ epidermal growth factor $\mathrm{ml}^{-1}$ (long EGF; E4269) and 10.9 ng fibroblast growth factor $\mathrm{ml}^{-1}$ (bovine FGF; F0291)) and incubated for $24 \mathrm{~h}$ at $39^{\circ} \mathrm{C}$ under a humidified atmosphere of $5 \% \mathrm{CO}_{2}$ in air. After maturation, the oocytes were washed twice in Hepes-buffered Tyrode's albumin-lactate-pyruvate (Hepes-TALP) and fertilization-TALP (Fert-TALP; Lu et al., 1987) supplemented with $10 \mu \mathrm{g}$ heparin $\mathrm{ml}^{-1}, 600 \mu \mathrm{g}$ penicillamine $\mathrm{ml}^{-1}$ and $220 \mu \mathrm{g}$ hypotaurine $\mathrm{ml}^{-1}$.

\section{In vitro fertilization and culture}

Spermatozoa were prepared from a frozen-thawed semen sample from a single sire of proven fertility. The semen was thawed in a waterbath at $37^{\circ} \mathrm{C}$, layered on to a discontinuous Percoll (Pharmacia Biotech, St Albans) gradient (45:90) and centrifuged at $2100 \mathrm{~g}$ for $25 \mathrm{~min}$. The pellet was resuspended in Hepes-TALP and centrifuged for a further $10 \mathrm{~min}$ at $1500 \mathrm{~g}$. The resulting motile sperm pellet was resuspended in Fert-TALP. The sperm suspension was adjusted to give a concentration of $1 \times$ $10^{6}$ spermatozoa $\mathrm{ml}^{-1}$ and added to fertilization wells each containing 45-65 oocytes, which had been washed twice in Hepes-TALP and Fert-TALP. Spermatozoa and oocyte complexes were co-incubated at $39^{\circ} \mathrm{C}$ under a humidified atmosphere of $5 \% \mathrm{CO}_{2}$ in air. After $18 \mathrm{~h}$, any remaining cumulus cells were removed by vortexing in Hepes-buffered synthetic oviductal fluid (SOF) supplemented with minimum essential and non-essential medium amino acids (Gibco), $1 \mathrm{mmol}$ glutamine $\mathrm{I}^{-1}$ and $4 \mathrm{mg} \mathrm{BSA} \mathrm{ml}{ }^{-1}$ (Hepes-SOFaaBSA; Tervit et al., 1972). The resultant putative zygotes were washed twice in Hepes-SOFaaBSA, placed in groups of 15 in $20 \mu \mathrm{l}$ droplets of SOFaaBSA under mineral oil and incubated at $39^{\circ} \mathrm{C}$ in a humidified atmosphere of $5 \% \mathrm{CO}_{2}, 5 \% \mathrm{O}_{2}$ and $90 \% \mathrm{~N}_{2}$.

\section{Immunosurgery}

Immunosurgery was performed on day 8 expanded blastocysts. The zonae pellucidae were removed from unhatched blastocysts by treatment with $0.5 \%(\mathrm{w} / \mathrm{v})$ pronase at $39^{\circ} \mathrm{C}$. The zona pellucida-free embryos were returned to culture medium for $2-3 \mathrm{~h}$ to recover before incubation in anti-bovine serum (B8270; 10\% (v/v) in Hepes-SOF). The embryos were washed in Hepes-SOF and incubated in guinea-pig complement serum (S1639; $25 \%(\mathrm{v} / \mathrm{v})$ in Hepes-SOF) supplemented with $0.01 \mathrm{mg}$ propidium iodide $\mathrm{ml}^{-1}$ at $39^{\circ} \mathrm{C}$. Treated blastocysts were transferred to individual $2 \mu \mathrm{l}$ droplets of Hepes-SOF and the ICM was removed from the lysed trophectoderm cells by aspiration through a narrow bore pipette.

\section{Trophectoderm biopsy}

Zona pellucida-free day 8 blastocysts were transferred to individual microdroplets of Hepes-SOF. Holding pipettes and sharp tipped glass needles were made from $1 \mathrm{~mm}$ glass capillary tubing with the aid of a microforge 
(Research Instruments, Penyrn) and siliconized with dimethyldichlorosilane solution to minimize cell adhesion to the glass. A narrow bore holding pipette, mounted on a micromanipulator (Leica, Milton Keynes), was used to maintain the blastocyst in position. Trophectoderm cell biopsies were obtained by free hand slicing through the mural trophectoderm using the sharp tip of a glass needle. The biopsies were returned to culture for $1 \mathrm{~h}$ to allow the trophectoderm cells to recover.

\section{Incubation of blastocysts, ICM and trophectoderm vesicles}

For the measurement of glucose and pyruvate uptake and lactate production, blastocysts, ICM and trophectoderm cells were transferred to $80 \mathrm{nl}$ droplets of SOFaaBSA, whereas for amino acid analysis, they were transferred to $1 \mu \mathrm{l}$ droplets of SOFaaBSA. All droplets were incubated under a mineral oil overlay in a humidified atmosphere of $5 \% \mathrm{CO}_{2}$ in air at $39^{\circ} \mathrm{C}$. For the measurement of carbohydrate metabolism, the duration of incubation was $30 \mathrm{~min}$ for intact blastocysts and 1 $\mathrm{h}$ for isolated ICM and trophectoderm vesicles. The production and consumption of amino acids were measured over $5 \mathrm{~h}$ for blastocysts, ICM and trophectoderm vesicles. Control droplets were incubated alongside the embryo-containing drops to account for any non-specific loss of nutrients.

\section{Counting of number of cells}

After incubation, blastocysts, isolated ICM and trophectoderm vesicles were transferred to microdroplets of SOFaaBSA supplemented with $1 \mathrm{mg}$ bisbenzimide $\mathrm{ml}^{-1}$ and cultured for $1 \mathrm{~h}$. They were removed, fixed in absolute ethanol, mounted individually in droplets of glycerol on a microscope slide and the cells were counted three times using a microscope (Leica).

\section{Micropuncturing technique}

The method for blastocyst micropuncturing and aspiration of blastocoel fluid was adapted from Brison et al. (1993). In brief, expanded day 8 blastocysts were removed from culture and transferred to a $10 \mathrm{nl}$ droplet of pre-warmed Hepes-SOFaaBSA under a mineral oil underlay. The blastocysts were immobilized by a holding pipette connected to an air-filled syringe and mounted on a micromanipulator. The medium surrounding the embryo was gently removed to ensure that the site of micropuncture was not contaminated by external culture medium. A finely pulled, oil-filled pipette was introduced through the mural trophectoderm to avoid damaging the ICM cells, and blastocoel fluid was aspirated gently until the blastocyst had fully collapsed around the pipette. The retrieved fluids were expelled into a dish under oil and frozen at $-80^{\circ} \mathrm{C}$ alongside $0.5 \mathrm{nl}$ control droplets of
SOFaaBSA. The pipettes for micropuncture and aspirating blastocoel fluid samples were made using a Pul-1 pipette puller (World Precision Instruments, Stevenage).

\section{Ultramicrofluorometric assays}

The concentrations of pyruvate, lactate and glucose in the blastocoel fluid and incubation droplets were measured non-invasively using ultramicrofluorometric techniques (Leese and Barton, 1984; Gardner and Leese, 1986). The changes in fluorescence were quantified using a Fluovert microscope (Leica) with photomultiplier and photometer attachments.

\section{Amino acid analysis}

Spent and control incubation droplets were diluted 1:40 in high performance liquid chromatography (HPLC) grade water (Fisher Scientific, Loughborough); pooled droplets of blastocoel fluid (2-7 nl) were diluted in $25 \mu \mathrm{l}$ water. The amino acid concentrations in the incubation droplets and blastocoel fluid were measured by reversephase HPLC as described previously (Lamb and Leese, 1994; Houghton et al., 2002) using a Kontron 500 Series automated HPLC system, with a Jasco F920 fluorescence detector and a $4.5 \times 250 \mathrm{~mm}$ Hypersil ODS-16 column (Jones Chromatography, Mid Glamorgan). Amino acid concentrations in experimental samples were evaluated by reference to control samples of known amino acid concentration. This method did not allow the detection of taurine, proline or cysteine.

\section{Statistical analysis}

Glucose and pyruvate consumption, and the production of lactate and amino acid consumption and production by blastocysts, ICM and trophectoderm vesicles were expressed as mean pmol per cell per $\mathrm{h} \pm \mathrm{SEM}$. Glycolytic index was determined as the proportion of glucose converted to lactate and was expressed as a percentage. The concentrations of glucose, pyruvate, lactate and amino acids in the blastocoel fluid were expressed as mean mmol $\mathrm{I}^{-1} \pm \mathrm{SEM}$. As small numbers of replicates were used in each batch, the data were compared using Kruskal-Wallis test. No significant differences between groups were recorded and so the different cohorts of blastocysts were pooled, before analysis by Student's $t$ test and one-way ANOVA with post hoc Fisher's least significant difference.

\section{Results}

Metabolic assessment of blastocysts, ICM and trophectoderm for glucose, lactate and pyruvate

The uptake of glucose and pyruvate, and production of lactate by whole blastocysts, isolated ICM and 
Table 1. Number of cells of intact bovine blastocysts, isolated inner cell mass (ICM) and trophectoderm vesicles

\begin{tabular}{lcccccc}
\hline & & \multicolumn{2}{c}{ ICM } & & \multicolumn{2}{c}{ Trophectoderm } \\
\cline { 3 - 5 } Cell population & Number of replicates & Number of cells & Proportion (\%) & & Number of cells & Proportion (\%) \\
\hline Blastocyst & 31 & $37.72 \pm 1.29$ & $31.19 \pm 0.37$ & $83.21 \pm 2.72$ & $68.81 \pm 0.37$ \\
Isolated ICM & 25 & $32.82 \pm 1.55$ & - & - & - \\
Isolated trophectoderm & 22 & - & - & $35.35 \pm 1.88$ & - \\
\hline
\end{tabular}

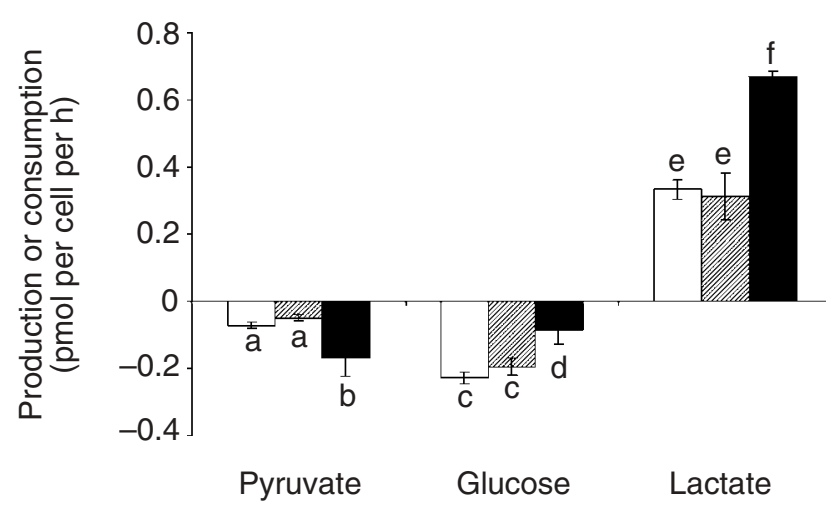

Fig. 1. Glucose and pyruvate consumption and lactate production by intact bovine blastocysts $(\square)$, isolated inner cell mass $(\square)$ and trophectoderm vesicles (ם). Data are expressed on a per cell basis \pm SEM. ${ }^{a-f}$ Different letters indicate significant differences between cell populations (lactate: $P<0.05$; glucose: $P<0.05$; pyruvate: $P<0.001)$.

trophectoderm are shown (Fig. 1). The metabolic profiles of the ICM and trophectoderm differed significantly. The trophectoderm showed a significantly higher pyruvate $(P<0.001)$ and lower glucose $(P<0.05)$ consumption, and an increased lactate $(P<0.05)$ production compared with the ICM on a per cell basis. The intact blastocyst profiles tended to reflect those of the isolated ICM rather than the trophectoderm.

The proportion of ICM cells in a day 8 expanded blastocyst was $31.19 \%$ compared with $68.81 \%$ for trophectoderm (Table 1). The metabolism of a composite blastocyst derived from the values for isolated ICM and trophectoderm metabolism and number of cells (Fig. 1 and Table 1) is shown (Fig. 2) alongside that of control blastocysts. Pyruvate consumption and lactate production were significantly higher in the composite blastocyst, whereas glucose consumption was significantly lower $(P<0.001)$. The glycolytic indices of isolated ICM and intact blastocyst were comparable (Fig. 3), but were markedly lower than those of the composite blastocyst and isolated trophectoderm $(P<0.001)$.

\section{Blastocoel fluid}

The concentrations of glucose, lactate and pyruvate in bovine blastocoel cavity and SOFaaBSA controls are

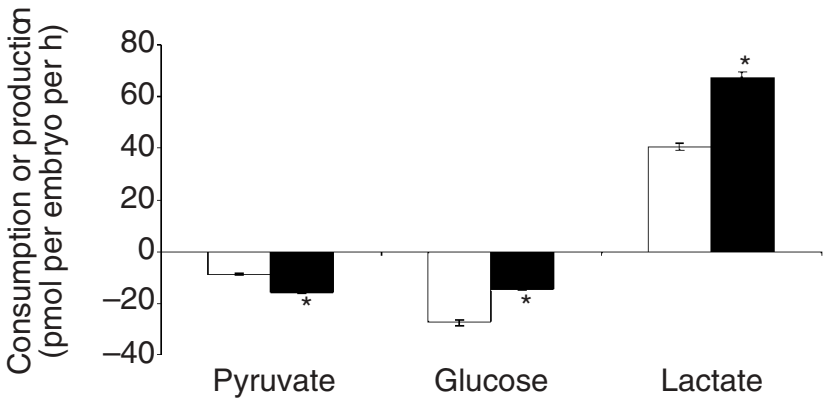

Fig. 2. The metabolism of a composite bovine blastocyst constructed on the basis of the data in Fig. 1 and Table 1, and of an intact blastocyst $(\square)$. * Denotes significant difference between intact and composite blastocysts $(P<0.001)$.

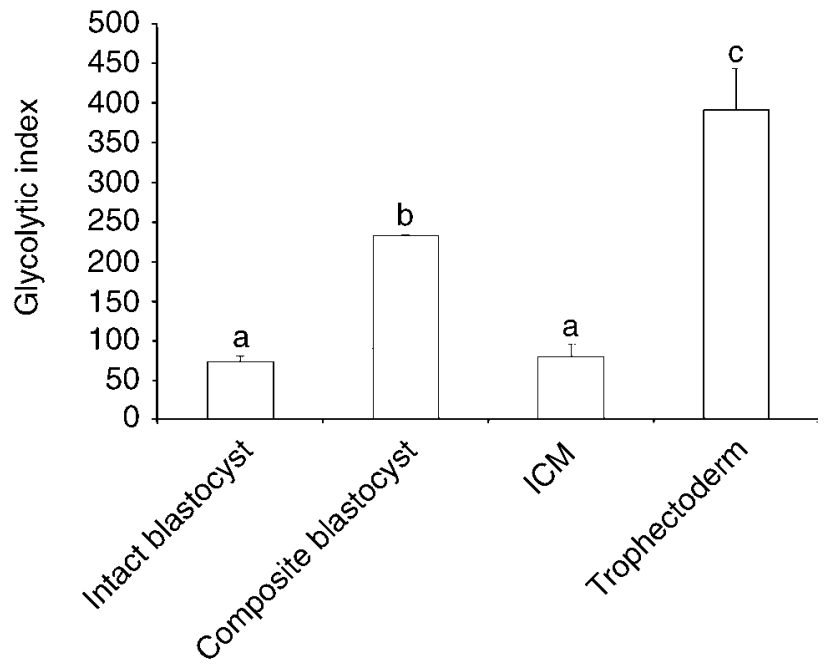

Fig. 3. Glycolytic index of intact and composite bovine blastocysts, and isolated inner cell mass (ICM) and trophectoderm. ${ }^{a-c}$ Different letters indicate significant differences $(P<0.001)$.

shown (Fig. 4). The concentration of lactate in blastocoel fluid was significantly higher, and pyruvate and glucose concentrations were significantly lower, than in SOFaaBSA culture medium.

Assessment of amino acids of blastocysts, ICM and trophectoderm

The production or consumption of amino acids from SOFaaBSA culture medium by intact blastocysts, isolated 
ICM and trophectoderm vesicles was measured using reverse-phase HPLC (Fig. 5). The uptake or production of all amino acids was significantly higher in the isolated cell lineages compared with intact blastocysts. All types of cell depleted aspartate, arginine and leucine, whereas alanine was produced consistently. Isolated ICM depleted asparagine, glycine, threonine, tyrosine, tryptophan and phenylalanine, whereas these were produced during the culture of trophectoderm vesicles. The reverse trend (that is, production in ICM culture and consumption in trophectoderm culture) was observed for methionine, valine, isoleucine, glutamate, serine, histidine and glutamine.

The production and consumption of amino acids by intact and composite blastocysts is shown (Fig. 6). Production of most of the amino acids increased in the composite blastocyst compared with the intact blastocyst; however, glutamate, histidine, glycine and threonine showed the opposite profile, that is, increased production in the intact blastocyst.

A summary of total amino acid production and consumption by intact blastocysts, ICM and trophectoderm vesicles is shown (Fig. 7). The isolated cell lineages have significantly increased total amino acid production and consumption profiles compared with intact blastocysts. This is reflected in total amino acid turnover (total production plus total consumption): blastocyst: 0.142 ; ICM: 1.21; and trophectoderm: 1.05 pmol per cell per h. Amino acid consumption values were higher for ICM than trophectoderm, but production values were similar. There was no difference in turnover between the ICM and trophectoderm $(P<0.05)$.

\section{Amino acid composition of blastocoel fluid}

A comparison of the concentrations of amino acids in bovine blastocoel fluid and SOFaaBSA is shown (Table 2). Most amino acids were present at higher concentrations in blastocoel fluid than in SOFaaBSA; however, only the concentrations of aspartate, glutamate, glycine, alanine and tryptophan were significantly higher. In contrast, concentrations of asparagine and threonine were significantly lower in blastocoel fluid than in SOFaaBSA.

\section{Discussion}

The energy-generating pathways available to the ICM and trophectoderm are the same as for somatic cells and include oxidative metabolism, notably of pyruvate, and the glycolytic conversion of glucose to lactate (Rieger et al., 1992; Thompson et al., 1996; Donnay and Leese, 1999). As only mural trophectoderm cells were isolated in the present study, the discussion will disregard any possible contribution of polar trophectoderm cells to intact blastocyst metabolism. The ICM had lower pyruvate consumption per cell than did the isolated

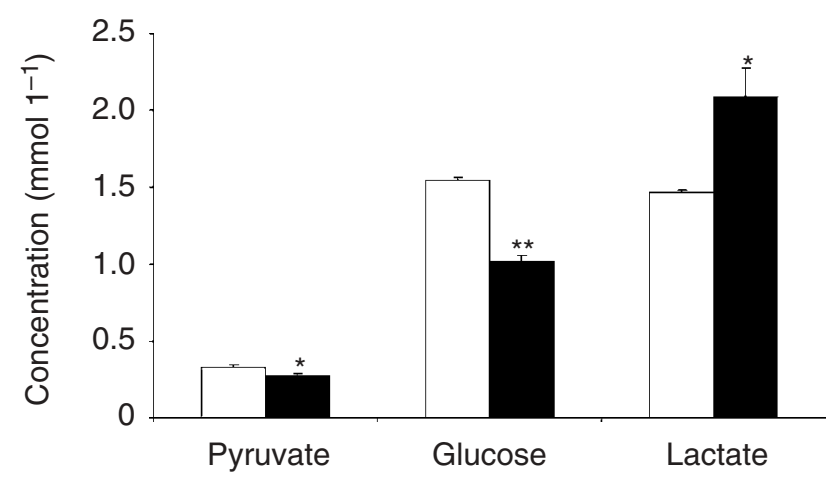

Fig. 4. Composition of blastocoel fluid in day 8 expanded bovine blastocysts $(\boldsymbol{\square}, n=8)$ and culture medium ( $\square, n=8$, Hepesbuffered synthetic oviductal fluid (SOF) supplemented with minimum essential and non-essential medium amino acids, $1 \mathrm{mmol}$ glutamine $\mathrm{I}^{-1}$ and $4 \mathrm{mg} \mathrm{BSA} \mathrm{m}^{-1}$ ). Asterisks denote significant differences between blastocoel fluid and culture medium $\left({ }^{*} P<0.05\right.$ and $\left.{ }^{* *} P<0.001\right)$

Table 2. Amino acid concentration in control synthetic oviductal fluid (SOF) supplemented with minimum essential and nonessential medium amino acids, 1 mmol glutamine $\mathrm{I}^{-1}$ and $4 \mathrm{mg}$ BSA ml-1 (control SOFaaBSA; $n=10$ ) and blastocoel fluid $(n=9)$

\begin{tabular}{lcc}
\hline Amino acid & $\begin{array}{c}\text { Control SOFaaBSA } \\
\left(\mathrm{mmol} \mathrm{I}^{-1}\right)\end{array}$ & $\begin{array}{c}\text { Blastocoel fluid } \\
\left(\mathrm{mmol} \mathrm{I}^{-1}\right)\end{array}$ \\
\hline Asp & $0.15 \pm 0.02$ & $5.62 \pm 0.26^{* *}$ \\
Glu & $0.15 \pm 0.00$ & $8.85 \pm 0.54^{* *}$ \\
Asn & $0.07 \pm 0.00$ & $0.03 \pm 0.02^{*}$ \\
Ser & $0.15 \pm 0.04$ & $0.62 \pm 0.07$ \\
His & $0.13 \pm 0.01$ & $0.42 \pm 0.02$ \\
Gln & $0.80 \pm 0.02$ & $0.86 \pm 0.05$ \\
Gly & $0.21 \pm 0.05$ & $2.16 \pm 0.51^{* *}$ \\
Thr & $2.67 \pm 0.26$ & $0.90 \pm 0.06^{* *}$ \\
Arg & $0.53 \pm 0.01$ & $0.93 \pm 0.08$ \\
Ala & $0.29 \pm 0.05$ & $2.97 \pm 0.49^{* *}$ \\
Tyr & $0.37 \pm 0.05$ & $0.55 \pm 0.10$ \\
Trp & $0.08 \pm 0.03$ & $0.48 \pm 0.20^{*}$ \\
Met & $0.11 \pm 0.03$ & $0.19 \pm 0.40$ \\
Val & $0.48 \pm 0.06$ & $0.71 \pm 0.15$ \\
Phe & $0.26 \pm 0.04$ & $0.53 \pm 0.14$ \\
Ile & $0.40 \pm 0.01$ & $0.64 \pm 0.13$ \\
Leu & $0.42 \pm 0.02$ & $0.91 \pm 0.06$ \\
Lys & $0.36 \pm 0.01$ & $0.53 \pm 0.04$ \\
\hline
\end{tabular}

Asterisks indicate significant differences compared with control SOFaaBSA group $\left({ }^{*} P<0.05\right.$ and $\left.{ }^{* *} P<0.001\right)$.

trophectoderm and intact blastocysts, which is indicative of relatively low oxidative metabolism. However, the glycolytic index of the ICM (79\%) indicated that $21 \%$ of the glucose consumed was fully oxidized, thereby possibly reducing the requirement for pyruvate consumption and oxidation. The metabolism of the isolated trophectoderm differed greatly from that of the ICM, and was characterized by higher pyruvate consumption, 


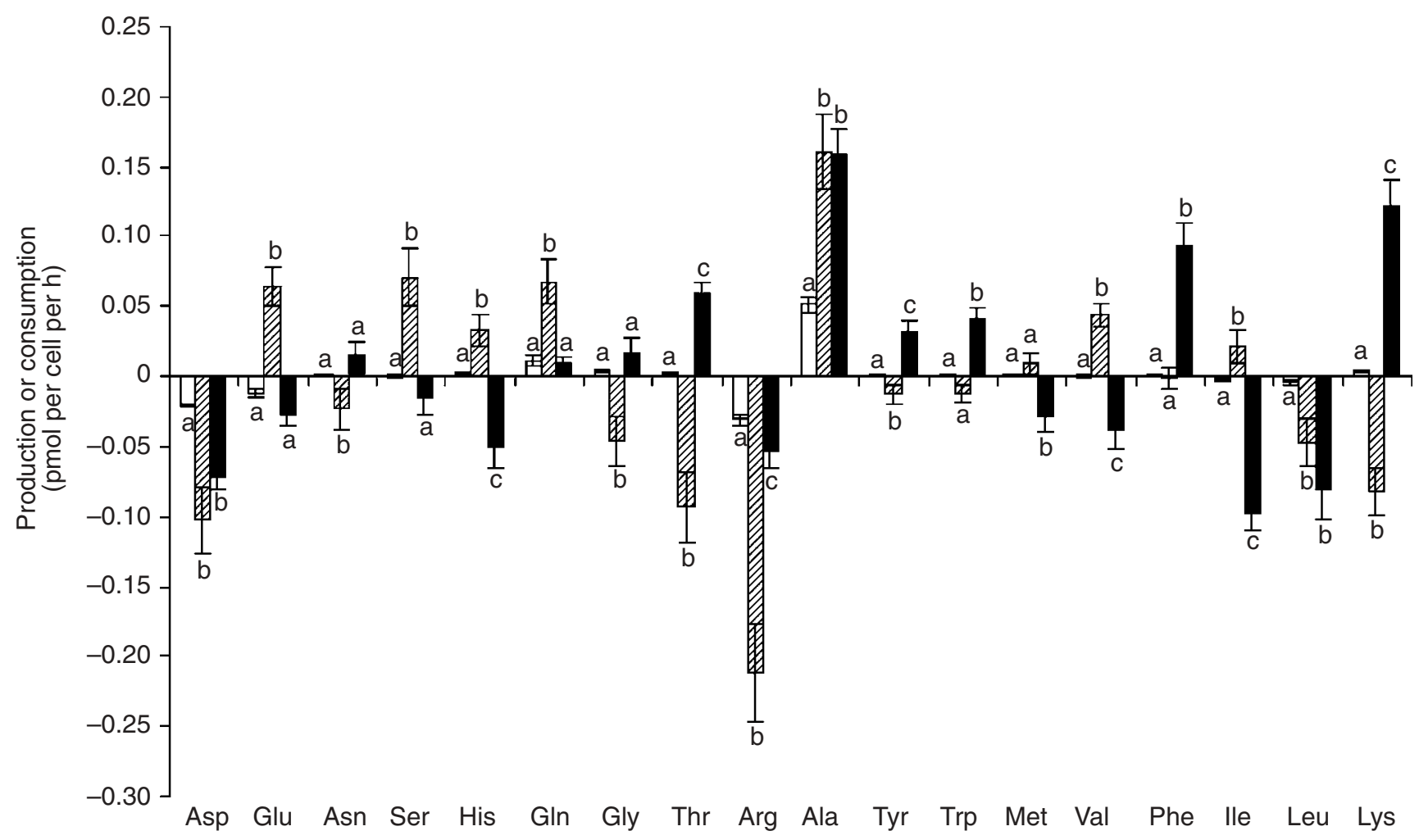

Fig. 5. Amino acid consumption or production by intact bovine blastocysts $(\square, n=28)$, isolated inner cell mass ( $\square, n=20)$ and trophectoderm vesicles $(\mathbf{\square}, n=14)$. ${ }^{\mathrm{a}-\mathrm{c}}$ For each amino acid, bars with different letters indicate significant differences between cell populations $(P<0.05)$.

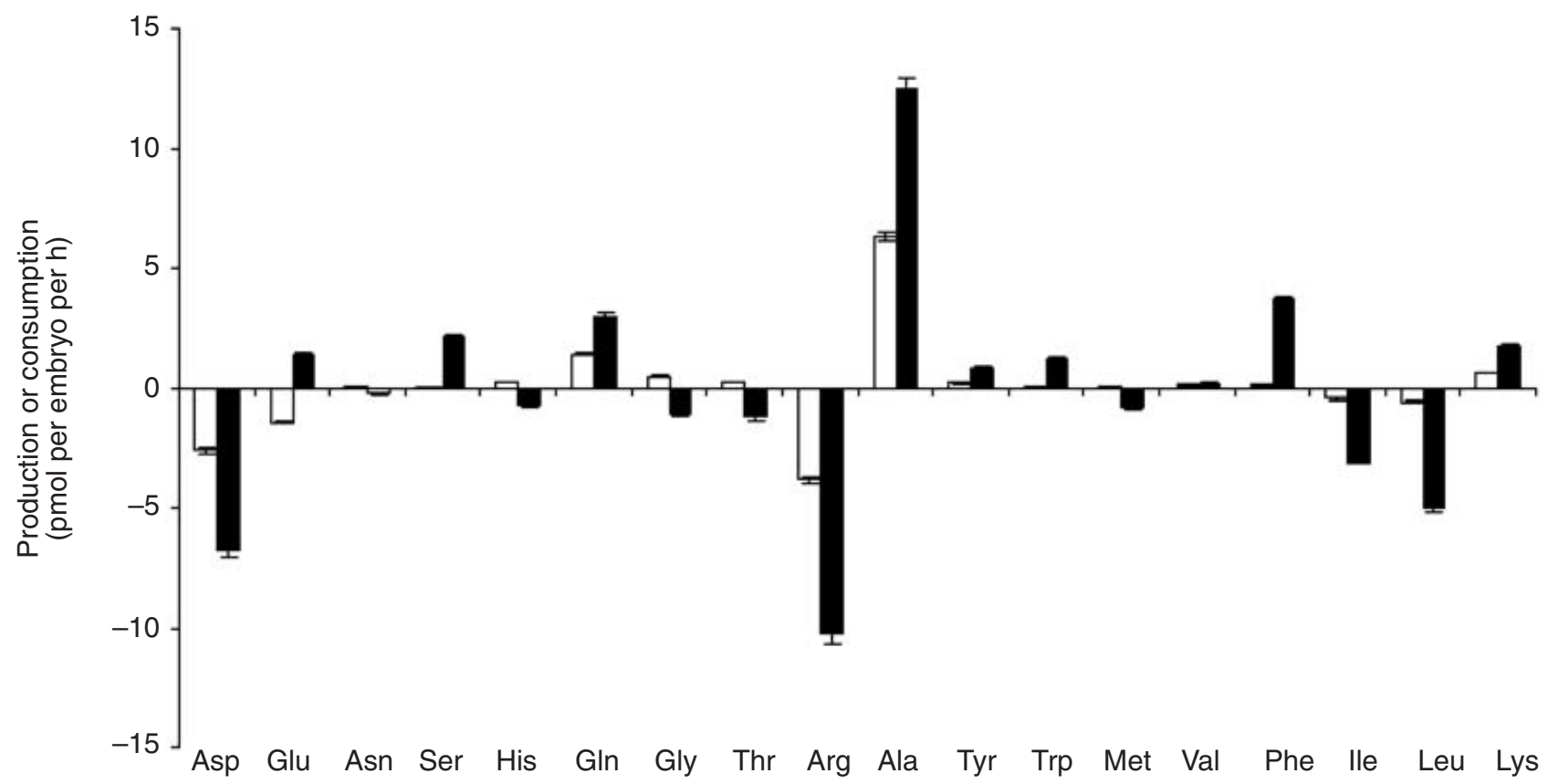

Fig. 6. Amino acid consumption or production by intact bovine blastocysts ( $\square$ ) and composite blastocysts ( $\mathbf{\square})$ constructed on the basis of the data in Table 1 and Fig. 5. The consumption or production of all amino acids differed significantly between composite and intact blastocysts $(P<0.001)$. 
lower glucose uptake and higher lactate output. The glycolytic index of the trophectoderm $(390 \%)$ indicated that all the glucose consumed was probably converted to lactate and that further lactate was derived from an additional source or sources. Two possibilities are by the conversion from pyruvate via lactate dehydrogenase $(\mathrm{LDH})$ or from amino acid catabolism, as occurs in the placenta (Bassett, 1991).

The pronounced difference in carbohydrate metabolism between the trophectoderm and ICM may reflect their diverging roles in embryological development. Upon hatching, the trophectoderm undergoes a period of rapid growth and elongation before attachment to the maternal caruncles. Enhanced glycolysis and, probably, glutaminolysis, best maintain an adequate provision of metabolic intermediates for biosynthetic pathways, as indicated by the metabolism of rapidly dividing cells in vitro (Newsholme et al., 1989; Leese, 1993; Wojtczak, 1996).

Amino acids improve the development of preimplantation mouse (Gardner and Lane, 1993), rat (Miyoshi et al., 1995), sheep (Gardner et al., 1994) and cow (Rosenkrans and First, 1994) embryos in vitro, and increase the number of cells in mouse blastocyst ICM (Lane and Gardner, 1997). Bovine embryos have a relatively low consumption and production of amino acids until the onset of genome activation, at the 8-16-cell stage (Frei et al., 1989), when there is a rapid increase, which continues throughout development to the blastocyst stage (Partridge and Leese, 1996; Jung et al., 1998).

Total amino acid turnover (that is, the sum of consumption and production) did not differ between isolated ICM and trophectoderm, but overall total consumption was significantly higher for the ICM than the trophectoderm. This finding indicates that the ICM may have a greater requirement for amino acids, perhaps due to differences in rates of protein synthesis (Handyside and Johnson, 1978) and cell division (Harlow and Quinn, 1982). In turn, this is likely to be a function of expression and localization of amino acid transporters (Miller and Schultz, 1985). Overall, the increased amino acid profile observed in the isolated cell lineages in the present study may reflect metabolic adaptation of trophectoderm and ICM to an artificial 'in vitro' culture environment (Morris et al., 2002) or a loss of metabolic control through the disruption of paracrine interactions upon isolation.

Certain amino acids consistently appeared (alanine and glutamine) or were depleted (aspartate, arginine and leucine) from the medium during incubation of trophectoderm and ICM. The production of alanine may result from amino nitrogen fixation, to prevent the accumulation of potentially toxic ammonium ions (Gardner and Lane, 1993; Partridge and Leese, 1996; Donnay and Leese, 1999). This may explain why all cell populations consistently produced alanine, that is, to eliminate ammonium.

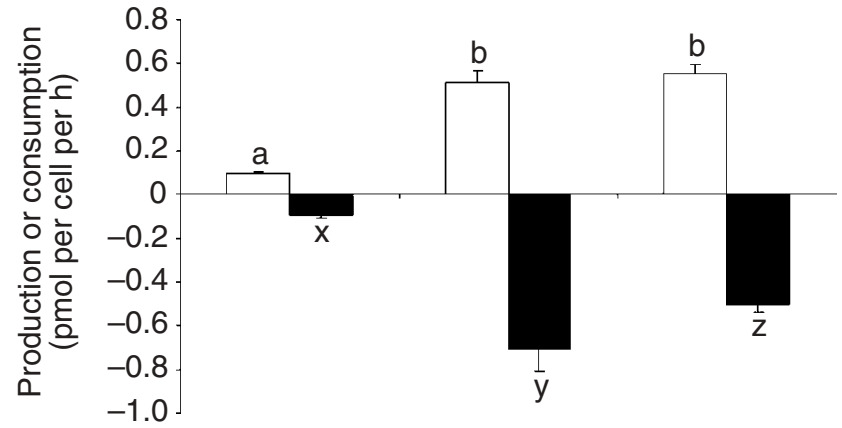

Blastocyst ICM Trophectoderm

Fig. 7. Total amino acid production ( $\square$ ) or consumption ( $\mathbf{\square}$ ) by intact bovine blastocysts, inner cell mass (ICM) and trophectoderm vesicles. ${ }^{a-c}$ or ${ }^{x-z}$ Different letters indicate significant differences between cell populations $\left({ }^{\mathrm{a}-\mathrm{c}} P<0.05\right.$ and $\left.{ }^{\mathrm{x}-\mathrm{z}} P<0.001\right)$.

Arginine and leucine were consistently depleted, in agreement with reports for in vivo and in vitro cattle blastocysts (Lee and Fukui, 1996; Partridge and Leese, 1996; Jung et al., 1998) and human embryos (Houghton et al., 2002). Leucine consumption may have resulted from its role as a signalling molecule that is capable of regulating gene expression, protein synthesis and blastocyst growth (Fox et al., 1998; Martin and Sutherland, 2001; Van Winkle, 2001). Similarly, arginine may be required for the production of nitric oxide, which regulates embryonic growth (Gouge et al., 1998). Van Winkle (2001) suggested that arginine and leucine might promote the growth of blastocysts by increasing nutrient supply and protein synthetic capacity via enhanced signalling. Therefore, the consistent consumption of these amino acids may indicate their requirement in blastocyst cells for gene regulation, cell signalling and protein synthesis.

Asparagine is incorporated into proteins, but is only taken up when de novo synthesis is insufficient to meet demand, for example in rapidly dividing cells such as malignant cells; this may account for the opposing profiles of asparagines, that is, consumption by the ICM and production by the trophectoderm.

The glucose and pyruvate uptake and lactate production values for the intact bovine blastocysts fell within the range reported in other studies (Thompson et al., 1996; Donnay and Leese, 1999), with the exception of glycolytic index; $73 \%$ in the present study and $100 \%$ in that of Thompson et al. (1996). The reasons for this may lie in minor differences in embryo production protocols, for example, the inclusion of amino acids in the fertilization medium used in the present study. Brief exposure to amino acid-free conditions has protracted effects on preimplantation embryos, and results in cultureinduced stress (Lane and Gardner, 1998).

There were significant differences between the metabolism of the intact and composite blastocysts, obtained by summing the consumption and production profiles of 
the isolated ICM and trophectoderm, and taking into account their representative numbers of cells. The composite blastocyst showed increased pyruvate consumption and lactate production with a decreased glucose uptake compared with that of the intact blastocyst. The intact blastocyst had a lower glycolytic index (73\%) than did the composite blastocyst (233\%). This lower index indicates that glucose may be metabolized more efficiently by the intact blastocyst in terms of ATP generation, with a greater reliance on oxidative metabolism, as upregulation of glycolysis is a marker of metabolic stress (Lane and Gardner, 1996; Leese et al., 1998). In contrast, Hewitson and Leese (1993) reported that metabolism of mouse composite blastocysts equalled that of intact blastocysts. These differences may be a consequence of differences in embryo glycolytic index between the two species. One possibility is that by virtue of their smaller volume mouse embryos are better able to satisfy their oxygen requirement in vitro, and therefore their capacity for aerobic respiration, than bovine embryos (Byatt-Smith et al., 1991).

When ICM and trophectoderm are isolated from the blastocyst, the paracrine signalling mechanisms governing their metabolism in situ may be lost, resulting in self-directed regulation. In addition, increased cultureinduced stress of both lineages may have occurred due to a loss of homeostatic regulation and protection within the intact blastocyst. The differences in metabolism may also be attributed to alterations in the microenvironment of the trophectoderm and the ICM. The apical and basolateral surfaces of trophectoderm cells are normally exposed to different environments (external and blastocoel fluid), whereas the ICM is normally exposed to a single specialized microenvironment, that is, blastocoel fluid.

Culture of in vivo- and in vitro-derived bovine embryos results in alanine production and arginine and leucine consumption (Lee and Fukui, 1996; Partridge and Leese, 1996; Jung et al., 1998; Kuran et al., 2002). A similar pattern was observed for both the intact and composite blastocysts. The composite blastocyst was metabolically more active in terms of amino acid turnover than the intact blastocyst, as it was with pyruvate, glucose and lactate, possibly reflecting alterations in trophectoderm and ICM metabolism due to a change in culture environment upon the loss of the blastocoel fluid.

The concentrations of pyruvate, glucose and lactate in blastocoel fluid differed significantly from those of the external medium. Pyruvate and glucose concentrations were lower in blastocoel fluid than in SOFaaBSA, whereas lactate concentration was higher. These results parallel data recorded in mice, rats and rabbits (Quinn and Wales, 1973; Brison et al., 1993), except that in rats, pyruvate concentration was higher in blastocoel fluid than externally (Brison et al., 1993). The lower pyruvate and glucose concentrations in bovine blastocoel fluid may be due to slow transport into the cavity, via facilitated transport or utilization by the ICM and trophectoderm or both (Brison et al., 1993; Hewitson and Leese, 1993).

The concentrations of substrates in blastocoel fluid are probably maintained within a range optimal for ICM development (Brison et al., 1993). In this respect, it has been demonstrated that high external concentrations of glucose inhibit ICM development within rat blastocysts (De Hertogh et al., 1991). The homeostatic mechanism used by preimplantation embryos to regulate substrate concentrations within the blastocoel cavity is dependent upon the expression and localization of glucose transporters (GLUTs). Pantaleon and Kaye (1998) suggested that the apical distribution of GLUT3 on trophectoderm membranes is responsible for the uptake of external glucose, which in combination with the basolaterally expressed GLUT1 provides glucose to the ICM. The localization of GLUT2 to membranes facing the blastocoel fluid may be indicative of a regulatory role in maintaining glucose concentration in the cavity; however, the precise mechanism remains to be determined.

Aspartate, glutamate, glycine and tryptophan were present at higher concentrations in blastocoel fluid compared with the external environment. However, the reverse was observed for threonine and asparagine concentrations, which were significantly lower in blastocoel fluid. It is possible that the high glutamate concentration within the cavity was due to its formation from $\alpha$ ketoglutarate as the final reaction in amino acid deamination. Alanine appears in increasing amounts at every stage of development of bovine preimplantation embryos (Partridge and Leese, 1996; Jung et al., 1998; Kuran et al., 2002). Hence, it is not surprising that it appears in such high concentrations within the blastocoel cavity $\left(2.9 \mathrm{mmol} \mathrm{I}^{-1}\right)$. In contrast, the concentrations of threonine, glutamine and asparagine were at lower or similar concentrations in the blastocoel to those of the external medium. A number of studies have revealed the presence of $\mathrm{Na}^{+}$-dependent and -independent transporters in the blastocyst. However, their specific localization in the ICM and trophectoderm, and role in determining the amino acid profile of blastocoel fluid, remain to be determined (Miller and Schultz, 1985; Jamshidi and Kaye, 1995; Van Winkle, 2001).

In conclusion, the intact blastocyst and isolated cell lineages differ markedly in their utilization and production of glucose, lactate, pyruvate and amino acids. Our data indicate that, upon isolation, the ICM and trophectoderm lose metabolic control, possibly through the absence of paracrine interactions or culture-induced cellular stress or both. This culminates in increased metabolic activity, as illustrated by the discrepancy between the 'intact' and 'composite' blastocysts.

This work was supported by a James Burgess Scholarship awarded to the University of York. 


\section{References}

Baltz JM (1993) Intracellular $\mathrm{pH}$ regulation in the early embryo BioEssays 15 523-530

Baltz JM (2001) Osmoregulation and cell volume regulation in the preimplantation embryo Current Topics in Developmental Biology 52 55-106

Bassett JM (1991) Symposium on 'recent research in the placenta' Proceedings of the Nutrition Society 50 311-319

Biggers JD, Bell JE and Benos DJ (1988) Mammalian blastocyst: transport functions in the developing epithelium American Journal of Physiology 255 C419-432

Brison DR and Leese HJ (1993) Role of chloride transport in the development of the rat blastocyst Biology of Reproduction 48 692-702

Brison DR, Hewitson LC and Leese HJ (1993) Glucose, pyruvate and lactate concentrations in the blastocoel cavity of rat and mouse embryos Molecular Reproduction and Development 35 227-232

Byatt-Smith JG, Leese HJ and Gosden RG (1991) An investigation by mathematical modelling of whether mouse and human preimplantation embryos in static culture can satisfy their demands for oxygen by diffusion Human Reproduction 6 52-57

De Hertogh R, Vanderheyden I, Pampfer S, Robin D, Dufrasne E and Delcourt J (1991) Stimulatory and inhibitory effects of glucose and insulin on rat blastocyst development in vitro. Diabetes 40 641-647

Donnay I and Leese HJ (1999) Embryo metabolism during the expansion of the bovine blastocyst Molecular Reproduction and Development 53 171-178

Ducibella T and Anderson E (1975) Cell shape and membrane changes in the eight-cell mouse embryo: prerequisites for morphogensis of the blastocyst Developmental Biology 47 45-58

Edirisinghe WR, Wales RG and Pike IL (1984) Studies of the distribution of glycogen between the inner cell mass and trophoblast cells of mouse embryos Journal of Reproduction and Fertility 71 533-538

Edwards LJ, Williams DA and Gardner DK (1998) Intracellular pH of the mouse preimplantation embryo: amino acids act as buffers of intracellular pH Human Reproduction 13 3441-3448

Epstein CJ and Smith SA (1973) Amino acid uptake and protein synthesis in preimplantation mouse embryos Developmental Biology 33 171-184

Fleming TP (1987) A quantitative analysis of cell allocation to trophectoderm and inner cell mass in the mouse blastocyst Developmental Biology 119 520-531

Fleming TP and Hay HJ (1991) Tissue-specific control of expression of the tight junction polypeptide ZO-1 in the mouse early embryo Development 113 295-304

Fox HL, Pham PT, Kimball SR, Jefferson LS and Lynch C (1998) Amino acid effects on translational repressor 4E-BP1 are mediated primarily by L-leucine in isolated adipocytes American Journal of Physiology 275 C1232-1238

Frei RE, Schultz GA and Church RB (1989) Qualitative and quantitative changes in protein synthesis occur at the 8-16 cell stage of embryogenesis in the cow Journal of Reproduction and Fertility 86 637-641

Gardner DK and Lane M (1993) Amino acids and ammonium regulate mouse embryo development in culture Biology of Reproduction 48377 385

Gardner DK and Leese HJ (1986) Non-invasive measurement of nutrient uptake by single cultured pre-implantation mouse embryos Human Reproduction 1 25-27

Gardner DK, Lane M, Spitzer A and Batt PA (1994) Enhanced rates of cleavage and development for sheep zygotes cultured to the blastocyst stage in vitro in the absence of serum and somatic cells: amino acids, vitamins, and culturing embryos in groups stimulate development Biology of Reproduction $\mathbf{5 0}$ 390-400

Gouge RC, Marshburn P, Gordon BE, Nunley W and Huet-Hudson YM (1998) Nitric oxide as a regulator of embryonic development Biology of Reproduction 58 875-879

Handyside AH and Johnson MH (1978) Temporal and spatial patterns of the synthesis of tissue-specific polypeptides in the preimplantation mouse embryo Journal of Embryology and Experimental Morphology 44 191199
Harlow GM and Quinn P (1982) Development of preimplantation mouse embryos in vivo and in vitro. Australian Journal of Biological Sciences 35 187-193

Hewitson LC and Leese HJ (1993) Energy metabolism of the trophectoderm and inner cell mass of the mouse blastocyst Journal of Experimental Zoology 267 337-343

Houghton FD, Hawkhead JA, Humpherson PG, Hogg JE, Balen AH, Rutherford AJ and Leese HJ (2002) Non-invasive amino acid turnover predicts human embryo developmental capacity Human Reproduction 17 999-1005

Jamshidi MB and Kaye PL (1995) Glutamine transport by mouse inner cell masses Journal of Reproduction and Fertility 104 91-97

Jaszczak S, Bentyn K and Choroszewska A (1972) Concentration of alphaamino nitrogen and free amino acid in the uterine and blastocoelic fluid of rabbit Acta Europaea Fertilitatis 3 183-191

Jung YG, Sakata T, Lee ES and Fukui Y (1998) Amino acid metabolism of bovine blastocysts derived from parthenogenetically activated or in vitro fertilized oocytes Reproduction, Fertility and Development 10 279-287

Kirchhof N, Carnwarth JW, Lemme E, Anastassiadis K, Scholer H and Niemann H (2000) Expression pattern of Oct-4 in preimplantation embryos of different species Biology of Reproduction 63 1698-1705

Kuran M, Robinson JJ, Brown DS and McEvoy TG (2002) Development, amino acid utilization and cell allocation in bovine embryos after in vitro production in contrasting culture systems Reproduction 124 155-165

Lamb VK and Leese HJ (1994) Uptake of a mixture of amino acids by mouse blastocysts Journal of Reproduction and Fertility 102 169-175

Lane M and Gardner DK (1996) Selection of viable mouse blastocysts prior to transfer using a metabolic criterion Human Reproduction 11 19751978

Lane M and Gardner DK (1997) Differential regulation of mouse embryo development and viability by amino acids Journal of Reproduction and Fertility 109 153-164

Lane M and Gardner DK (1998) Amino acids and vitamins prevent cultureinduced metabolic perturbations and associated loss of viability of mouse blastocysts Human Reproduction 13 991-997

Lee ES and Fukui Y (1996) Synergistic effect of alanine and glycine on bovine embryos cultured in a chemically defined medium and amino acid uptake by in vitro-produced bovine morulae and blastocysts Biology of Reproduction 55 1383-1389

Leese HJ (1991) Metabolism of the preimplantation embryo Oxford Reviews of Reproduction 13 35-72

Leese HJ (1993) Energy metabolism in preimplantation development. In Preimplantation Embryo Development pp 72-82 Ed. BD Bavister. Springer-Verlag, London

Leese HJ (1995) Metabolic control during preimplantation mammalian development Human Reproduction Update 1 63-72

Leese HJ and Barton AM (1984) Pyruvate and glucose uptake by mouse ova and preimplantation embryos Journal of Reproduction and Fertility $\mathbf{7 2}$ 9-13

Leese HJ, Donnay I and Thompson JG (1998) Human assisted conception: a cautionary tale. Lessons from domestic animals Human Reproduction 13 Supplement 4 184-202

Lindenbaum A (1973) A survey of naturally occurring chelating ligands Advances in Experimental Medical Biochemistry 40 67-77

Lu KH, Gordon I, Gallagher M and McGovern H (1987) Pregnancy established in cattle by transfer of embryos derived from in vitro fertilisation of oocytes matured in vitro. Veterinary Record 121259 260

Martin PM and Sutherland AE (2001) Exogenous amino acids regulate trophectoderm differentiation in the mouse blastocyst through an mTORdependent pathway Developmental Biology 240 182-193

Miller JG and Schultz GA (1985) Amino acid transport in mouse blastocyst compartments Journal of Embryology and Experimental Morphology 89 149-158

Miyoshi K, Abeydeera LR, Okuda K and Niwa K (1995) Effects of osmolarity and amino acids in a chemically defined medium on development of rat one-cell embryos Journal of Reproduction and Fertility 103 27-32 
Morris DG, Humpherson PG, Leese HJ and Sreenan JM (2002) Amino acid turnover by elongating cattle blastocysts recovered on days 14-16 after insemination Reproduction 124 667-673

Newsholme EA, Newsholme P, Curi R, Crabtree B and Ardawi MSM (1989) Glutamine metabolism in different tissues; its physiological and pathological importance. In Perspectives in Human Nutrition pp 71-98 Eds JM Kinney and PR Borum. Urban \& Schwarzenberg, Balitmore

Pantaleon M and Kaye PL (1998) Glucose transporters in preimplantation development Reviews of Reproduction 3 77-81

Partridge RJ and Leese HJ (1996) Consumption of amino acids by bovine preimplantation embryos Reproduction, Fertility and Development 8 945-950

Pratt HP (1985) Membrane organization in the preimplantation mouse embryo Journal of Embryology and Experimental Morphology 90101 121

Quinn P and Wales RG (1973) The in vitro metabolism of (U-14C) glucose by the preimplantation rabbit embryo Australian Journal of Biological Sciences 26 653-656

Rieger D, Loskutoff NM and Betteridge KJ (1992) Developmentally related changes in the uptake and metabolism of glucose, glutamine and pyruvate by cattle embryos produced in vitro. Reproduction, Fertility and Development 4 547-557

Robinson DH and Benos DJ (1991) Glucose metabolism in the trophectoderm and inner cell mass of the rabbit embryo Journal of Reproduction and Fertility 91 493-499
Rosenkrans CF, Jr and First NL (1994) Effect of free amino acids and vitamins on cleavage and developmental rate of bovine zygotes in vitro. Journal of Animal Science 72 434-437

Slager HG, Lawson KA, van den Eijnden-van Raaij AJ, de Laat SW and Mummery CL (1991) Differential localization of TGF-beta2 in mouse preimplantation and early postimplantation development Developmental Biology 145 205-218

Tervit HR, Whittingham DG and Rowson LE (1972) Successful culture in vitro of sheep and cattle ova Journal of Reproduction and Fertility 30 493-497

Thompson JG, Partridge RJ, Houghton FD, Cox CI and Leese HJ (1996) Oxygen uptake and carbohydrate metabolism by in vitro derived bovine embryos Journal of Reproduction and Fertility 106 299-306

Van Winkle LJ (2001) Amino acid transport regulation and early embryo development Biology of Reproduction 64 1-12

Watson AJ and Barcroft LC (2001) Regulation of blastocyst formation Frontiers in Bioscience 6 D708-730

Wojtczak L (1996) The crabtree effect: a new look at an old problem Acta Biochimica Polonica 42 361-368

Received 5 February 2003.

First decision 5 March 2003

Revised manuscript received 24 April 2003.

Accepted 28 May 2003. 